\title{
Effect of overexpressing $n h a A$ and nhaR on sodium tolerance and lactate production in Escherichia coli
}

\author{
Xianghao Wu', Ronni Altman², Mark A Eiteman ${ }^{1 *}$ and Elliot Altman²
}

\begin{abstract}
Background: Like other bacteria, Escherichia coli must carefully regulate the intracellular concentration of sodium ion $\left(\mathrm{Na}^{+}\right)$. During the bacterial production of any organic acid, cations like $\mathrm{Na}^{+}$invariably accumulate during a process which must maintain a near neutral $\mathrm{pH}$. In this study, the $E$. coli nhaA gene encoding the $\mathrm{Na}^{+} / \mathrm{H}^{+}$antiporter membrane protein and the $n h a R$ gene encoding the NhaA regulatory protein were overexpressed in wild-type $E$. coli MG1655 and in MG1655 pflB (ALS1317) which lacks pyruvate formate lyase activity and thus accumulates lactate under anaerobic conditions.

Results: Expression of either the nhaA or nhaR gene on the high copy inducible expression vector pTrc99A caused a significant reduction in the growth rate of MG1655. No change in growth rate was observed for MG1655 or ALS1317 for $\mathrm{Na}^{+}$concentrations of $0.75-0.90 \mathrm{M}$ when the medium copy pBR322 plasmid was used to overexpress the two genes. In a fed-batch process to produce the model acid lactate with $\mathrm{NaOH}$ addition for $\mathrm{pH}$ control, lactate accumulation ceased in MG1655, MG1655/pBR322, MG1655/pBR322-nhaR and MG1655/pBR322-nhaA when the concentration reached 55-58 g/L. In an identical process lactate accumulation in MG1655/pBR322-nhaAR did not terminate until the concentration reached over $70 \mathrm{~g} / \mathrm{L}$.

Conclusions: Although overexpression the genes did not improve growth rate at high $\mathrm{Na}^{+}$concentrations, the overexpression of nhaA and nhaR together led to a 25\% increase in lactate production. Thus, the observed (absence of) impact that these genetic modifications had on growth rate is a poor indicator of their effect on acid accumulation. The overexpression of nhaAR did not cause faster lactate production, but permitted the culture to continue accumulating lactate at $10 \%$ greater $\mathrm{Na}^{+}$concentration.
\end{abstract}

Keywords: Sodium tolerance, nhaA, nhaR, Lactate, Lactic acid, Fed-batch fermentation

\section{Background}

The sodium ion $\left(\mathrm{Na}^{+}\right)$plays an important role in cellular function and bioenergetics, and cells rely on a very efficient homeostatic mechanism to maintain the intracellular sodium concentration [1]. E. coli typically maintains a sodium gradient across the cell membrane $\left(\mathrm{Na}_{\text {in }}^{+}<\mathrm{Na}_{\text {out }}^{+}\right)$ via two $\mathrm{Na}^{+} / \mathrm{H}^{+}$antiporter membrane proteins, NhaA and $\mathrm{NhaB}[2,3]$. These antiporters exchange $\mathrm{Na}^{+}$or $\mathrm{Li}^{+}$for $\mathrm{H}^{+}$ $[4,5]$ and are driven by the electrochemical proton gradient (i.e., $\mathrm{H}_{\text {out }}^{+}>\mathrm{H}_{\text {in }}^{+}$) generated by the primary proton pumps. NhaA encoded by nhaA [6] is absolutely required

\footnotetext{
* Correspondence: eiteman@engr.uga.edu

'BioChemical Engineering Program, College of Engineering, University of Georgia, Athens, GA 30602, USA

Full list of author information is available at the end of the article
}

for survival in the presence of $700 \mathrm{mM} \mathrm{Na}^{+}$at a pH of 6.8 [2]. NhaB alone confers limited sodium tolerance to cells, though this protein becomes essential when the lack of NhaA expression limits growth [7].

During exponential growth, nhaA responds to $\mathrm{Na}^{+}$by increasing transcription via a $\mathrm{Na}^{+}$-specific regulatory system mediated by the positive regulator protein NhaR encoded by $n h a R$ [8]. The expression of nhaR, which is located just downstream of $n h a A$, is induced specifically by $\mathrm{Na}^{+}$. The NhaR protein regulates $n h a A$ expression by undergoing a conformational change upon $\mathrm{Na}^{+}$binding, which modifies the NhaR-nhaA contact points [8]. Hence, NhaR appears to be both a sensor and a transducer of the $\mathrm{Na}^{+}$signal.

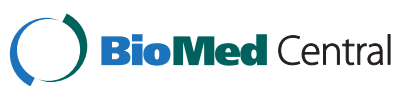


The accumulation of cations like $\mathrm{Na}^{+}$is germane for bioprocesses which generate an organic acid. If an optimal $\mathrm{pH}$ is to be maintained for the continued formation of such products, a base such as $\mathrm{NaOH}$ must be added into the system leading to the accumulation of cations. Previous results which show that addition of the osmoprotectant betaine improves organic acid accumulation [9] suggest that organic acid accumulation in the model organism E. coli may be limited by the accumulation of cation rather than by the acid anion itself. Acid accumulation is readily achieved by $E$. coli through a $p f B$ knockout which lacks pyruvate formate lyase activity and although unable to grow, generates lactate under anaerobic conditions [10]. The objective of this study was to examine the effect of overexpressing nhaA and nhaR on growth and lactate production in $E$. coli.

\section{Results}

Overexpression of nhaA or nhaR using the high-copy inducible expression vector pTrc99A

The NhaA protein serves as $\mathrm{a} \mathrm{Na}^{+}$extruding antiporter, while NhaR serves as a positive regulator for the expression of the $n h a A$ gene. We first wanted to test whether increased expression of $n h a A$ or $n h a R$ would increase the maximum specific growth rate of $E$. coli in the presence of high $\mathrm{Na}^{+}$ concentration. Although previous studies have considered the effect of $n h a A$ overexpression (only) on growth [11,12], these studies used complex media, focused on changes in the promoter to affect expression level, and did not explicitly measure growth rate at both low and high $\mathrm{Na}^{+}$concentration. We examined the effects of overexpressing nhaA and $n h a R$ in the widely used pTrc99A high-copy number inducible expression vector, which contains the origin of replication from pUC8 [13].

First, we determined how IPTG induction affected the growth rates of MG1655/pTrc99A-nhaA and MG1655/ pTrc99A-nhaR in the defined medium without additional $\mathrm{NaCl}$ (less than $0.10 \mathrm{M}$ total $\mathrm{Na}^{+}$concentration) and in the presence of $0.75 \mathrm{M} \mathrm{NaCl}$. The wild-type strain MG1655 attained a growth rate of about $0.69 \mathrm{~h}^{-1}$ in low $\mathrm{NaCl}$ and $0.17 \mathrm{~h}^{-1}$ in $0.75 \mathrm{M} \mathrm{NaCl}$. In the absence of IPTG induction, MG1655/pTrc99A-nhaA attained similar growth rates and MG1655/pTrc99A-nhaR about 20\% lower growth rates compared to the wild-type. However, induction of protein expression proved very detrimental to the growth rate of both strains (Figure 1). Even in the absence of additional $\mathrm{NaCl}$, induction of pTrc99A-nhaA prevented growth when the IPTG concentration was above $40 \mu \mathrm{M}$. Moreover, the growth rates were relatively sensitive to IPTG concentration.

\section{Overexpression of nhaA and nhaAR using the medium- copy plasmid pBR322}

Because overexpression of $n h a A$ or $n h a R$ from pTrc99A induced lethality and was very sensitive to expres-

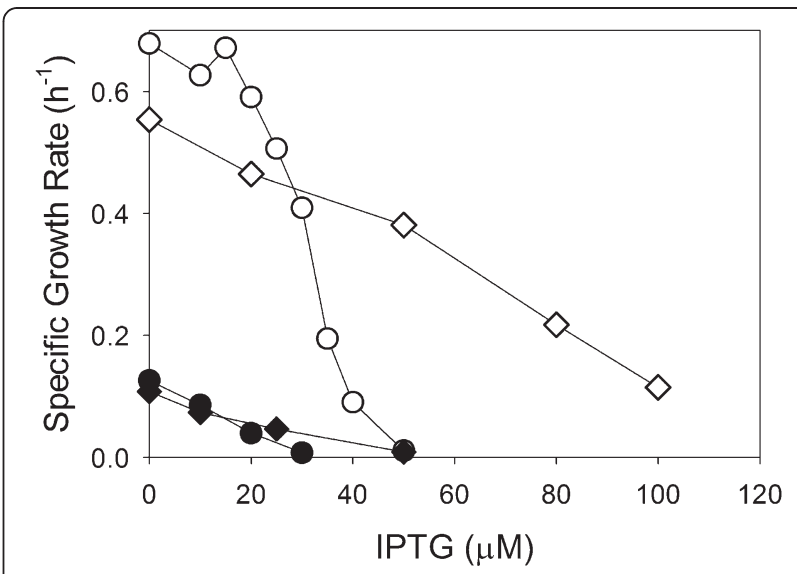

Figure 1 Maximum specific growth rate ( $\mu_{M A X}$ ) of MG1655/ pTrc99A-nhaA $(O, \bullet)$ and MG1655/pTrc99A-nhaR $(\diamond, \diamond)$ at low $(<0.10 \mathrm{M}) \mathrm{Na}^{+}$concentration (hollow symbols) and at $0.75 \mathrm{M} \mathrm{Na}^{+}$concentration (solid symbols). The specific growth rate of $\mathrm{MG} 1655$ was about $0.69 \mathrm{~h}^{-1}$ with $<0.10 \mathrm{M} \mathrm{Na}^{+}$ and $0.17 \mathrm{~h}^{-1}$ at $0.75 \mathrm{M} \mathrm{Na}^{+}$concentration.

sion level-an observation similarly noted by other researchers in the case of $n h a A[11,12]$-we cloned nhaA, $n h a R$ and $n h a A R$ including the relevant promoter(s) into the promoterless pBR322 cloning vector, which exists at about 20 copies per cell [14]. In growth rate experiments with each of the five constructs (MG1655, MG1655/ pBR322, MG1655/pBR322-nhaA, MG1655/pBR322-nhaR and MG1655/pBR322-nhaAR) using a defined medium we found that the maximum specific growth rate decreased with increasing $\mathrm{Na}^{+}$concentration (Figure 2). No difference was observed in the maximum specific growth rate for three of the strains over this concentration range (MG1655, MG1655/pBR322-nhaA, MG1655/pBR322-nhaAR), while MG1655/pBR322 and MG1655/pBR322-nhaR showed about $30 \%$ lower growth rates. None of the five strains were able to grow in this defined medium containing $0.91 \mathrm{M} \mathrm{Na}$ ${ }^{+}$. However, expression of $n h a A$ and $n h a R$ under these conditions was not detrimental to cell growth in contrast to expression using pTrc99A, even with greatly reduced inducer concentration. Quantitative real time polymerase chain reaction (qPCR) analysis of mRNA levels using both the $\beta$-lactamase bla gene from pBR322 and the elongation factor Tu tufA gene from the chromosome as controls showed that the mRNA levels of nhaA increased 8.4-fold in pBR322-nhaA and 15.3-fold in pBR322-nhaAR with respect to the chromosomal levels, while the mRNA levels of nhaR increased 10.5-fold in pBR322-nhaR and 29.9-fold in pBR322-nhaAR with respect to the chromosomal levels. Interestingly, no increase in $n h a A$ mRNA levels was observed in pBR322-nhaR. 


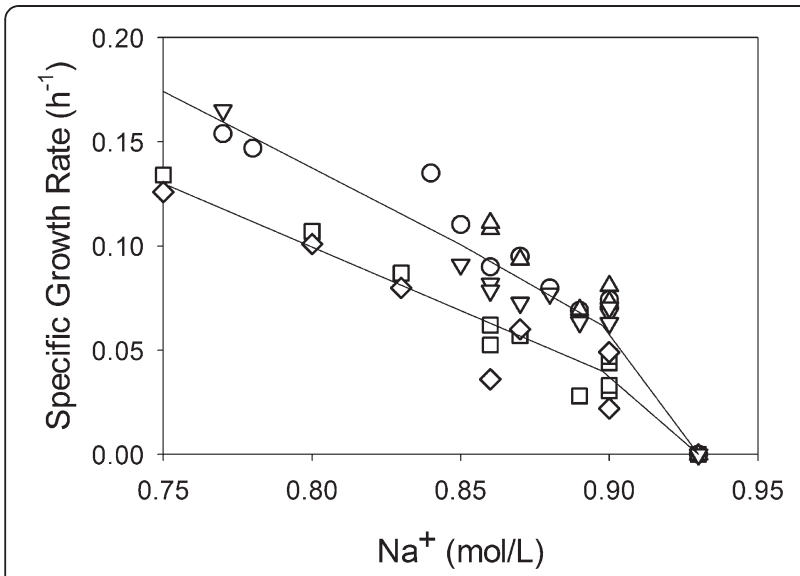

Figure 2 Maximum specific growth rate $\left(\mu_{\mathrm{MAX}}\right)$ of MG1655 (O), MG1655/pBR322 ( $\square$ ), MG1655/pBR322-nhaA ( $\Delta$ ), MG1655/ pBR322-nhaR $(\diamond)$, and MG1655/pBR322-nhaAR $(\nabla)$ with increasing total $\mathrm{Na}^{+}$concentration.

\section{Lactate production}

Although overexpression of nhaA, nhaR or nhaAR using the pBR322 plasmid did not confer increased $\mathrm{Na}^{+}$tolerance to $E$. coli based on maximum specific growth rate, a beneficial effect of overexpressing these genes might be manifested in other ways. For example, a culture of $E$. coli must be supplied with large quantities of a neutralizing base such as $\mathrm{NaOH}$ when the culture accumulates an organic acid. The accumulation of $\mathrm{Na}^{+}$during this process could reduce the rate of organic acid synthesis even though growth might not be affected or might not even be desired to generate the acid product. Since $E$. coli with a knockout of the $p f l B$ gene encoding pyruvate formate lyase will readily accumulate lactate [10], we next examined the possibility that overexpression of $n h a A$, nhaR or nhaAR allowed for greater accumulation of this model acid in pflB strains which would not be growing when lactate accumulated. Since pyruvate formate lyase activity is destroyed under aerobic conditions [15], the $p f l B$ knockout would not be expected to have any impact on aerobic cell growth.

To study the effect of nhaA, nhaR or nhaAR overexpression on lactate accumulation, five constructs were compared including two controls-the pflB knockout ALS1317 without the plasmid and ALS1317/pBR322, as well as the $p f l B$ knockout containing either nhaA, nhaR or nhaAR (ALS1317/pBR322-nhaA, ALS1317/pBR322-nhaR, or ALS1317/pBR322-nhaAR). We first confirmed that overexpression of nhaA, nhaR or nhaAR in ALS1317 had no affect on growth rate compared to MG1655 carrying the identical plasmids (data not shown). Then, these strains were compared with controlled fed-batch bioprocesses in which a low glucose concentration was automatically maintained, and the processes were terminated when glucose was no longer being consumed. The two control strains ALS1317 and ALS1317/pBR322 each achieved 55$56 \mathrm{~g} / \mathrm{L}$ lactate (Table 1). Similarly, for ALS1317/pBR322nhaA or ALS1317/pBR322-nhaR overexpressing nhaA or $n h a R$ alone, the process was automatically terminated when the concentration reached $58 \mathrm{~g} / \mathrm{L}$ or less. However, in ALS1317/pBR322-nhaAR overexpressing both nhaA and $n h a R$, the culture continued to consume glucose until the lactate concentration reached over $70 \mathrm{~g} / \mathrm{L}$. Interestingly, the succinate concentration attained was also greater for ALS1317/pBR322-nhaAR than for the other constructs: 9.7 g/L versus 3.4-6.3 g/L. The higher total acid concentration was not attained at a greater rate; the lactate productivity for all five different constructs was essentially identical in the range $1.42-1.45 \mathrm{~g} / \mathrm{L} \cdot \mathrm{h}$. Instead, the presence of both nhaA and nhaR permitted the prolonged production of lactate. Also, the final $\mathrm{Na}^{+}$concentration for ALS1317/ pBR322-nhaAR was about $10 \%$ greater than for the other constructs.

\section{Discussion}

In this study using E. coli, maximum specific growth rate was initially presumed to serve as an indicator for salt "tolerance", and growth rate was carefully measured in defined medium over several doubling times. Previous studies have generally used less quantified measures and complex media. Also, previous studies have often involved the $n h a B$ gene, encoding for a secondary $\mathrm{Na}^{+}$ transporter protein. For example, in order to isolate the gene conferring increased $\mathrm{Na}^{+} / \mathrm{H}^{+}$antiporter activity, merely growth $(+)$ or no growth $(-)$ was reported for strains in the presence of $0.1 \mathrm{M} \mathrm{Li}^{+}$[16]. Padan et al. measured doubling times of $E$. coli in Lysogeny Broth (LB) containing low $(0.08 \mathrm{M})$ and high $(0.7 \mathrm{M}) \mathrm{Na}^{+}$of the K12 strain TA15, the $\triangle$ nhaA derived strain NM81, and NM81 overexpressing nhaA [2]. They reported a five-fold increase in doubling time (i.e., a five-fold decrease in growth rate) in the nhaA knockout, but insignificant difference between TA15 and NM81-nhaA ${ }^{+}$. Pinner et al. studied doubling times of both $n h a A$ and $n h a B$ knockouts as functions of $\mathrm{pH}$ and found $E$. coli nhaA nhaB to be very sensitive to $\mathrm{Na}^{+}$, with growth ceasing at $50 \mathrm{mM}$ [7]. The focus of each of these studies

Table 1 Lactate production and final $\mathrm{Na}^{+}$concentration in fed-batch fermentation

\begin{tabular}{|c|c|c|c|}
\hline Strain & $\frac{\text { Lactate }}{(\mathrm{g} / \mathrm{L})}$ & $\frac{\text { Succinate }}{(g / L)}$ & $\frac{\text { Final }\left[\mathrm{Na}^{+}\right]}{(\mathrm{mol} / \mathrm{L})}$ \\
\hline ALS1317 & $54.5(1.8)$ & $4.9(1.3)$ & $0.87(0.00)$ \\
\hline ALS1317/pBR322 & $56.0(2.5)$ & $6.0(0.3)$ & $0.99(0.02)$ \\
\hline ALS1317/pBR322-nhaA & $57.6(0.1)$ & $6.3(0.3)$ & $0.98(0.01)$ \\
\hline ALS1317/pBR322-nhaR & $55.7(2.3)$ & $3.4(0.3)$ & $0.82(0.01)$ \\
\hline ALS1317/pBR322-nhaAR & $71.2(0.8)$ & $9.7(3.7)$ & $1.09(0.05)$ \\
\hline
\end{tabular}

Values indicate mean of replicate fermentations (standard deviations). 
was the decrease in growth rate as a result of knockouts in $n h a A$ and/or $n h a B$ and the relative importance of these genes in $\mathrm{Na}^{+}$tolerance, and not whether an elevated growth rate could be obtained by overexpressing either nhaA or nhaB.

Our studies were based on the simple hypothesis that careful overexpression of $n h a A$ and/or nhaR should allow E. coli, under conditions of greater $\mathrm{Na}^{+}$concentration, to attain a higher growth rate and to accumulate more acid. However, our studies showed elevated expression of $n h a A$, $n h a R$ or nhaAR did not improve growth rate as a function of $\mathrm{Na}^{+}$concentration. Our results contrast a similar report with Zymomonas mobilis showing that elevated nhaA expression permitted this strain to grow in complex medium with $0.195 \mathrm{M}$ sodium acetate, whereas no growth was observed using the wild-type control [17]. Expression of the E. coli nhaA gene in rice enhanced this plant's salt tolerance as measured by germination rate, average growth rate of the stem, shoot weight, root weight and rice yield per plant [18]. Also, the E. coli nhaA gene expressed in Saccharomyces cerevisiae significantly improved yeast growth on agar plates in the presence of $\mathrm{Li}^{+}$but had no effect in the presence of $\mathrm{Na}^{+}$[19], while overexpression of the sod2 gene in Schizosaccharomyces pombe increased $\mathrm{Na}^{+}$tolerance as measured by average diameter of colonies on Agar medium [20]. One possible explanation for our results is that while NhaA did enhance $\mathrm{Na}^{+}$extrusion, the growth rate of E. coli in defined medium was limited not by extracellular $\mathrm{Na}^{+}$concentration, but by another mechanism such as simple membrane integrity or DNA replication.

Although growth rate was not affected by nhaA and/ or nhaR overexpression, and overexpression of nhaA or nhaR alone in E. coli with a pflB knockout did not enhance lactate accumulation compared to controls in a fed-batch fermentation, overexpression of both nhaA and $n h a R$ permitted $25 \%$ greater lactate accumulation. Because we incorporated the complete regulatory region, including both the P1 $\sigma^{70}$ nhaR-dependent promoter and the P2 $\sigma^{38}$ nhaR-independent promoter of wild-type nhaA, which allows nhaA to be expressed under a variety of physiological conditions [21], in the pBR322$n h a A$ and pBR322-nhaAR plasmids, our observation that pBR322-nhaAR allows increased lactate accumulation, whereas pBR322-nhaA does not, clearly demonstrates the importance of NhaR in sustaining lactate accumulation by $E$. coli in the presence of increasing $\mathrm{Na}^{+}$. Since NhaA is positively regulated by NhaR [22], the specific effect of NhaR could be simply due to the different amounts of NhaA $\mathrm{Na}^{+} / \mathrm{H}^{+}$antiporter protein produced in these two constructs. Indeed, qPCR results showed greater expression of both nhaA and nhaR in pBR322nhaAR compared to either pBR322-nhaA or pBR322$n h a R$. Although there is a basal presence of NhaA in wild-type E. coli, nhaA expression depends on a functional $n h a R$ gene [23]. Thus, without the elevated expression of $n h a R$, the level of NhaA produced by pBR322-nhaA may be insufficient to allow for increased lactate accumulation. The failure of merely overexpressing nhaA or nhaR via the high-copy plasmid pTrc99A demonstrates the rigid control necessary for the production of the NhaA membrane protein. Importantly, our results do not preclude an alternative role for NhaR which may be unrelated to NhaA: for example, this gene may impact membrane proteins which are involved in lactate permeation. Nevertheless, elevated levels of both NhaA and NhaR were necessary for enhanced lactate accumulation.

\section{Conclusions}

Overexpression of $n h a A$ or $n h a R$ had no impact on E. coli $\mathrm{Na}^{+}$tolerance as measured by specific growth rate, but the combination of both nhaA and nhaR did allow E. coli to accumulate a $25 \%$ higher concentration of lactate. Such a marked increase in final acid titer may benefit the production of other high-volume commercially relevant acids in E. coli. The regulation accomplished by nhaR, whether to nhaA or to some other unknown gene or protein, is therefore critical in allowing cells to $\mathrm{Na}^{+}$tolerance during organic acid generation. Specific growth rate, however, appears be a poor indicator of microbial 'tolerance' in the context of its correlation with the accumulation of a biochemical product.

\section{Methods}

\section{Strains and growth medium}

Strains used in this study are listed in Table 2. To construct ALS1317, a P1vir lysate was prepared from NZN111 [24], and the pflB:: Cam deletion was transduced into MG1655 and chloramphenicol resistant transductant colonies were selected.

MG1655 genomic DNA was used as a template with $P f u$ DNA polymerase, and primers were designed based on the published E. coli MG1655 genome sequence [26] (GenBank accession number U00096). The nhaA coding sequence resides at bases 17,489 through 18,655 on the

\section{Table 2 Strains used in this study}

\begin{tabular}{|c|c|c|}
\hline Strains/Plasmids & Genotype & Notes \\
\hline MG1655 & E. coli F- $\lambda$ - ilvG rfb-50 rph-1 & Wild type \\
\hline ALS1317 & MG1655 pflB:: Cam & This study \\
\hline pTrc99A & $\mathrm{Amp}^{\mathrm{R}} \operatorname{trcPO} \mid \mathrm{lac}^{\mathrm{Q}}$ ColE1 ori & [13] \\
\hline pTrc99A-nhaA & Amp $^{R}$ trcPO lacl ${ }^{Q}$ ColE1 ori nhaA & This study \\
\hline pTrc99A-nhaR & Amp $^{R}$ trcPO lacl ${ }^{Q}$ ColE1 ori nhaR & This study \\
\hline pBR322 & Amp $^{R}$, Tet ${ }^{R}$, ColE1 replicon & {$[25]$} \\
\hline pBR322-nhaA & Amp $^{R}$, ColE1 replicon nhaA & This study \\
\hline pBR322-nhaAR & Amp $^{R}$, ColE1 replicon nhaAR & This study \\
\hline
\end{tabular}


E. coli chromosome while the nhaR coding sequence resides just downstream of $n h a A$ at bases 18,715 through 19,620. To construct pTrc99A-nhaA, the forward primer $5^{\prime}$ TACTATGGTACCCAGGAGAACAG CTATGAAACATCTGCATCGATTCTTTAGC 3 ', which introduced a $K p n I$ restriction site and Shine Dalgarno ribosome binding site just before the start codon of nhaA, thus converting the original GTG start codon to an ATG start codon, and the reverse primer 5'AGAGAGAGAGAGAGA GAG AGAAGCTTTAACAA TGAAAAGGGAGCCGTTTATG $3^{\prime}$, which introduced a HindIII restriction site immediately downstream of the TGA stop codon of $n h a A$, were utilized. The regions of homology to the MG1655 genome are underlined. The amplified 1,265 bp fragment was digested with $K p n \mathrm{I}$ and HindIII and ligated into pTrc99A, which had been digested with the same two restriction enzymes. To construct pTrc99A-nhaR, the forward primer 5'TACTAT GGTACCTTAGGAGGTAACAGCTATGAGCATGTCT CATATCAATTACAAC $3^{\prime}$ which introduced a KpnI restriction site and Shine Dalgarno ribosome binding site just before the ATG start codon and the reverse primer 5'ACACACACACACAAACAACCTGCAGTCGTGGTA ATGTACAACTAATCTATCT $3^{\prime}$, which introduced a PstI restriction site immediately downstream of the TAA stop codon, were utilized. The regions of homology to the MG1655 genome are underlined. The amplified 1,016 bp fragment was digested with $K p n \mathrm{I}$ and PstI and ligated into $\mathrm{p} \operatorname{Trc} 99 \mathrm{~A}$, which had been digested with the same two restriction enzymes.

As with pTrc99A-nhaA and pTrc99A-nhaR, to construct pBR322-nhaA, pBR322-nhaR and pBR322-nhaAR, MG1655 genomic DNA was used as a template with $P f u$ DNA polymerase, and primers were designed based on the published E. coli MG1655 genome sequence. To construct pBR322-nhaA, bases 17,229 through 18,658 of the MG1655 chromosome were amplified using the forward primer 5'TACTTTATGAATTCCATCGCCGAC TGACAACAAA $3^{\prime}$ and reverse primer 5'TACTTTATC CAAGGCTGTCAAACTGATGGACGCA 3 '. 261 bases upstream of the start of the nhaA coding sequence were included in the amplified region to incorporate both the P1 and P2 promoters for nhaA. Dover and Padan showed that nhaA is transcribed by both P1, a $\sigma^{70}$ nhaRdependent promoter and $\mathrm{P} 2$, a $\sigma^{38}$ nhaR-independent promoter and that these promoters are both used under different physiological conditions [21].

An EcoRI restriction site was introduced at the beginning of the forward primer, while a StyI restriction site was introduced at the end of the reverse primer. The regions of homology to the MG1655 genome are underlined. To construct pBR322-nhaR, bases 17,931 through 19,787 of the MG1655 chromosome were amplified using the forward primer 5'TACTTTATGAATTCT
TCCGTTAGCGCTGAAGATC $3^{\prime}$ and reverse primer 5'TACTTTATCCAAGGCTACTCTTTTCAGCATCCCC $3^{\prime} .784$ bases upstream of the start of the nhaR coding sequence were included in the amplified region to incorporate the promoter for $n h a R$. This region was shown by Carmel et al. to contain the promoter for nhaR [27]. An EcoRI restriction site was introduced at the beginning of the forward primer, while a StyI restriction site was introduced at the end of the reverse primer. The regions of homology to the MG1655 genome are underlined.

To construct pBR322-nhaAR, bases 17,229 through 19,787 of the MG1655 chromosome were amplified using the forward primer 5'TACTTTATGAATTCCA TCGCCGACTGACAACAAA $3^{\prime}$ and reverse primer 5'TACTTTATCCAAGGCTACTCTTTTCAGCATCCCC $3^{\prime}$. Just as with pBR322-nhaA, 261 bases upstream of the start of the nhaA coding sequence were included in the amplified region to incorporate both the P1 and P2 promoters for nhaA in pBR322-nhaAR. An EcoRI restriction site was introduced at the beginning of the forward primer, while a StyI restriction site was introduced at the end of the reverse primer. The regions of homology to the MG1655 genome are underlined. Both PCR products were gel isolated, digested with EcoRI and StyI, and ligated into the pBR322 plasmid which had been digested with the same two restriction enzymes.

The defined medium contained (per L): 10 g glucose, $1.70 \mathrm{~g}$ citric acid, $13.30 \mathrm{~g} \mathrm{KH}_{2} \mathrm{PO}_{4}, 4.50 \mathrm{~g}\left(\mathrm{NH}_{4}\right)_{2} \mathrm{HPO}_{4}$, $1.2 \mathrm{~g} \mathrm{MgSO}_{4} \cdot 7 \mathrm{H}_{2} \mathrm{O}, 13 \mathrm{mg} \mathrm{Zn}\left(\mathrm{CH}_{3} \mathrm{COO}\right)_{2} \cdot 2 \mathrm{H}_{2} \mathrm{O}$ $1.5 \mathrm{mg} \mathrm{CuCl} \cdot 2 \mathrm{H}_{2} \mathrm{O}, 15 \mathrm{mg} \mathrm{MnCl}_{2} \cdot 4 \mathrm{H}_{2} \mathrm{O}, 2.5 \mathrm{mg}$ $\mathrm{CoCl}_{2} \cdot 6 \mathrm{H}_{2} \mathrm{O}, 3.0 \mathrm{mg} \mathrm{H}_{3} \mathrm{BO}_{3}, 2.5 \mathrm{mg} \mathrm{Na} \mathrm{MoO}_{4} \cdot 2 \mathrm{H}_{2} \mathrm{O}$, $100 \mathrm{mg} \mathrm{Fe}(\mathrm{III})$ citrate, $4.5 \mathrm{mg}$ thiamine $\cdot \mathrm{HCl}$, and $8.4 \mathrm{mg}$ $\mathrm{Na}_{2}($ EDTA $) \cdot 2 \mathrm{H}_{2} \mathrm{O}$. All cultures had an initial $\mathrm{pH}$ of 7.0 and were grown in $250 \mathrm{~mL}$ baffled shake flasks at $37^{\circ} \mathrm{C}$ and $250 \mathrm{rpm}$ (19 mm pitch). $\mathrm{Na}^{+}$tolerance was quantified by growing strains in this medium to an optical density (OD) of 2.0, and then transferring the culture into a series of flasks containing the defined medium with additional $\mathrm{NaCl}$ to achieve the desired $\mathrm{Na}^{+}$concentration. In these second shake flasks the optical density was measured 5-7 times during exponential growth to calculate the maximum specific growth rate.

\section{qPCR analysis of mRNA levels}

The quantification of $n h a A$ and nhaR mRNA levels from cells containing the various pBR322 constructs was determined using $\mathrm{qPCR}$ with the $\beta$-lactamase bla gene from pBR322 and the elongation factor Tu tufA gene from the chromosome serving as controls. Total RNA was prepared from each pBR322 construct and quantified and then complementary DNA (cDNA) was prepared using RNA primers designed to initiate replication as close to the end of the coding sequence as possible. Amplicons were designed to be approximately 
$100 \mathrm{bp}$ and forward primers were designed to initiate replication as close to the start codon of the coding sequence as possible. For $n h a A$ the 5 ACUGAUGGACGC AAACGAAC 3'RNA primer was used for CDNA synthesis, while the forward 5'TCTTTAGCAGTGATGCCTCG 3 'and reverse 5'TCGTGATACCATCCACTGGT 3'DNA primers were used to generate a $100 \mathrm{bp}$ amplicon. For nhaR the 5'UUAACGCACCGCUGGACUAA 3'RNA primer was used for cDNA synthesis, while the forward $5^{\prime}$ AAAGAAGGTTCCGTGGTTGG $3^{\prime}$ and reverse $5^{\prime}$ TA ATTTGCCTTGCAGGCGCT 3'DNA primers were used to generate a $102 \mathrm{bp}$ amplicon. For bla the 5'UGC UUAAUCAGUGAGGCACC 3'RNA primer was used for CDNA synthesis, while the forward 5'TTCAA CATTTCCGTGTCGCC $3^{\prime}$ and reverse 5'CCCAACTG ATCTTCAGCATC $3^{\prime}$ DNA primers were used to generate a $109 \mathrm{bp}$ amplicon. For tufA the 5'GAACUUUA GCAACAACGCCC 3 'RNA primer was used for CDNA synthesis, while the forward 5'TGAACGTACAAAACC GCACG $3^{\prime}$ and reverse $5^{\prime}$ GTAGGTTTTAGCCAGTAC GG 3'DNA primers were used to generate a $103 \mathrm{bp}$ amplicon.

\section{Fed-batch processes}

For lactate production, each of the five constructs (ALS1317, ALS1317/pBR322, ALS1317/pBR322-nhaA, ALS1317/pBR322-nhaR, or ALS1317/pBR322-nhaAR) was first grown in a $250 \mathrm{~mL}$ shake flask containing $50 \mathrm{~mL}$ of medium. After $12 \mathrm{~h}$ the contents were used to inoculate a $2.5 \mathrm{~L}$ bioreactor (Bioflo 310, New Brunswick Scientific Co., New Brunswick, NJ, USA) containing $1.0 \mathrm{~L}$ of medium with $20 \mathrm{~g} / \mathrm{L}$ glucose. During an initial aerobic phase of about $6 \mathrm{~h}$ the agitation was maintained at $400 \mathrm{rpm}$, and air and $\mathrm{O}_{2}$ were mixed as necessary at a $1.0 \mathrm{~L} / \mathrm{min}$ total flow rate to maintain the dissolved oxygen above $40 \%$ of saturation until the OD reached 8.0. During a second, anaerobic phase the agitation was maintained at $200 \mathrm{rpm}$, and a 9:1 mixture of $\mathrm{N}_{2}$ and $\mathrm{CO}_{2}$ was sparged at $0.5 \mathrm{~L} / \mathrm{min}$. During this phase the glucose concentration was maintained at $2-4 \mathrm{~g} / \mathrm{L}$ using a $600 \mathrm{~g} / \mathrm{L}$ glucose solution automatically fed in response to the measurement of an on-line glucose analyzer (YSI 2700 SELECT, YSI Life Sciences Inc., Yellow Springs, $\mathrm{OH}, \mathrm{USA})$. Each fed-batch experiment was terminated when the glucose solution was not demanded for $3 \mathrm{~h}$. For both phases, the $\mathrm{pH}$ was controlled at 7.0 using $30 \%(\mathrm{w} / \mathrm{v})$ $\mathrm{NaOH}$, and the temperature at $37^{\circ} \mathrm{C}$. All fermentations were run in duplicate.

\section{Analytical methods}

The optical density at $600 \mathrm{~nm}$ (OD) (UV-650 spectrophotometer, Beckman Instruments, San Jose, CA, USA) was used to monitor cell growth. Concentrations of soluble organic compounds were determined by high performance liquid chromatography as previously described [28].

\section{Competing interests}

The authors declare that they have no competing interests.

\section{Authors' contributions}

ME and EA conceived the study. XW, RA, ME and EA designed components of the study and interpreted the results. RA completed the molecular biology aspects of this work. XW carried out the fermentations and acquired the data. XW prepared the manuscript draft. All authors read and approved the final manuscript.

\section{Acknowledgments}

The authors acknowledge Sarah A. Lee for assistance with growth studies.

\section{Author details}

${ }^{1}$ BioChemical Engineering Program, College of Engineering, University of Georgia, Athens, GA 30602, USA. ²Department of Biology, Middle Tennessee State University, Murfreesboro, TN 37132, USA.

Received: 19 April 2012 Accepted: 25 January 2013

Published: 29 January 2013

\section{References}

1. Padan E, Krulwich TA: Sodium stress. In Bacterial stress responses. Edited by Storz G, Hengge-Aronis R. Washington, DC: American Society for Microbiology; 2000:117-130.

2. Padan E, Maisler N, Taglicht D, Karpel R, Schuldiner S: Deletion of ant in Escherichia coli reveals its function in adaptation to high salinity and an alternative $\mathrm{Na}^{+} / \mathrm{H}^{+}$antiporter system(s). J Biol Chem 1989, 264:20297-20302.

3. West IC, Mitchell P: Proton/sodium ion antiport in Escherichia coli. Biochem J 1974, 44:87-90.

4. Pinner $\mathrm{E}$, Padan $\mathrm{E}$, Schuldiner $\mathrm{S}$ : Cloning, sequencing, and expression of the $n h a B$ gene, encoding a $\mathrm{Na}^{+} / \mathrm{H}^{+}$antiporter in Escherichia coli. J Biol Chem 1992, 267:11064-11068.

5. Taglicht D, Padan E, Schuldiner S: Proton-sodium stoichiometry of NhaA, an electrogenic antiporter from Escherichia coli. J Biol Chem 1993, 268:5382-5387.

6. Niiya S, Yamasaki K, Wilson TH, Tsuchiya T: Altered cation coupling to melibiose transport in mutants of Escherichia coli. J Biol Chem 1982, 257:8902-8906.

7. Pinner E, Kotler Y, Padan E, Schuldiner S: Physiological role of NhaB, a specific $\mathrm{Na}^{+} / \mathrm{H}^{+}$antiporter in Escherichia coli. J Biol Chem 1993, 268:1729-1734.

8. Carmel O, Rahav-Manor O, Dover N, Shaanan B, Padan E: The $\mathrm{Na}^{+}$-specific interaction between the LysR-type regulator, NhaR, and the nhaA gene encoding the $\mathrm{Na}^{+} / \mathrm{H}^{+}$antiporter of Escherichia coli. EMBO J 1997, 16:5922-5929

9. Zhu Y, Eiteman MA, Altman R, Altman E: High glycolytic flux improves pyruvate production by a metabolically engineered Escherichia coli strain. Appl Environ Microbiol 2008, 74:6649-6655.

10. Zhu J, Shimizu K: The effect of pfl gene knockout on the metabolism for optically pure D-lactate production by Escherichia coli. Appl Microbiol Biotechnol 2004, 64:367-375.

11. Taglicht D, Padan E, Schuldiner S: Overproduction and purification of a functional $\mathrm{Na}+/ \mathrm{H}+$ antiporter coded by nhaA (ant) from Escherichia coli. J Biol Chem 1991, 266:11289-11294.

12. Inoue H, Noumi T, Shimomura T, Takimoto N, Tsuchiya T, Kanazawa H: pHdependent growth retardation of Escherichia coli caused by overproduction of $\mathrm{Na}+/ \mathrm{H}+$ antiporter. Biol Pharm Bull 1998, 21:1128-1133

13. Amann $\mathrm{E}$, Ochs $\mathrm{B}$, Abel $\mathrm{KJ}$ : Tightly regulated tac promoter vectors useful for the expression of unfused and fused proteins in Escherichia coli. Gene 1988, 69:301-315.

14. Covarrubias L, Cervantes L, Covarrubias A, Soberón X, Vichido I, Blanco A, Kupersztoch-Portnoy YM, Bolivar F: Construction and characterization of new cloning vehicles. V. Mobilization and coding properties of pBR322 and several deletion derivatives including pBR327 and pBR328. Gene 1981, 13:25-35. 
15. Knappe J, Neugebauer FA, Blaschkowski HP, Gänzler M: Post-translational activation introduces a free radical into pyruvate formate-lyase. Proc Natl Acad Sci USA 1984, 81:1332-1335

16. Karpel R, Olami Y, Taglicht D, Schuldiner S, Padan E: Sequencing of the gene ant which affects the $\mathrm{Na}+/ \mathrm{H}+$ antiporter activity in Escherichia coli. J Biol Chem 1988, 263:10408-10414.

17. Yang S, Land ML, Klingeman DM, Pelletier DA, Lu TYS, Martin SL, Guo HB, Smith JC, Brown SD: Paradigm for industrial strain improvement identifies sodium acetate tolerance loci in Zymomonas mobilis and Saccharomyces cerevisiae. Proc Natl Acad Sci 2010, 107:10395-10400.

18. Wu L, Fan Z, Guo L, Li Y, Chen Z, Qu L: Over-expression of the bacterial nhaA gene in rice enhances salt and drought tolerance. Plant Sci 2004, 168:297-302.

19. Ros R, Montesinos C, Rimon A, Padan E, Serrano R: Altered $\mathrm{Na}^{+}$and $\mathrm{Li}^{+}$ homeostasis in Saccharomyces cerevisiae cells expressing the bacterial cation antiporter NhaA. J Bacteriol 1998, 180:3131-3136.

20. Jia ZP, McCullough N, Martel R, Hemmingsen S, Young PG: Gene amplification at a locus encoding a putative $\mathrm{Na}^{+} / \mathrm{H}^{+}$antiporter confers sodium and lithium tolerance in fission yeast. EMBO J 1992 11:1631-1640

21. Dover $\mathrm{N}$, Padan E: Transcription of $n h a A$, the main $\mathrm{Na}^{+} / \mathrm{H}^{+}$antiporter of Escherichia coli, is regulated by $\mathrm{Na}^{+}$and growth phase. J Bacteriol 2001, 183:644-653.

22. Rahav-Manor O, Carmel O, Karpel R, Taglicht D, Glaser G, Schuldiner S, Padan E: NhaR, a protein homologous to a family of bacterial regulatory proteins (LysR), regulates nhaA, the sodium proton antiporter gene in Escherichia coli. J Biol Chem 1992, 267:10433-10438.

23. Dover $\mathrm{N}$, Higgins CF, Carmel O, Rimon A, Pinner E, Padan E: $\mathrm{Na}^{+}$-induced transcription of nhaA, which encodes an $\mathrm{Na}^{+} / \mathrm{H}^{+}$antiporter in Escherichia coli, is positively regulated by nhaR and affected by hns. J Bacteriol 1996, 178:6508-6517.

24. Bunch PK, Mat-Jan F, Lee N, Clark DP: The IdhA gene encoding the fermentative lactate dehydrogenase of Escherichia coli. Microbiol 1997 143:187-195.

25. Bolivar F, Rodriguez RL, Greene PJ, Betlach MC, Heyneker HL, Boyer HW, Crosa JH, Falkow S: Construction and characterization of new cloning vehicles. II. A multipurpose cloning system. Gene 1977, 2:95-113.

26. Blattner FR, Plunkett $G$, Bloch CA, Perna NT, Burland V, Riley M, ColladoVides J, Glasner JD, Rode CK, Mayhew GF, Gregor J, Davis NW, Kirkpatrick HA, Goeden MA, Rose DJ, Mau B, Shao Y: The complete genome sequence of Escherichia coli K-12. Science 1997, 277:1453-1462.

27. Carmel O, Dover N, Rahav-Manor O, Dibrov P, Kirsch D, Karpel R, Schuldiner $\mathrm{S}$, Padan E: A single amino acid substitution (Glu134 $\rightarrow$ Ala) in NhaRI increases the inducibility by $\mathrm{Na}+$ of the product of nhaA, a Na+/H + antiporter gene in Escherichia coli. EMBO J 1994, 13:1981-1989.

28. Eiteman MA, Chastain MJ: Optimization of the ion-exchange analysis of organic acids from fermentation. Anal Chim Acta 1997, 338:69-75.

doi:10.1186/1754-1611-7-3

Cite this article as: Wu et al:: Effect of overexpressing nhaA and nhaR on sodium tolerance and lactate production in Escherichia coli. Journal of Biological Engineering 2013 7:3.

\section{Submit your next manuscript to BioMed Central and take full advantage of:}

- Convenient online submission

- Thorough peer review

- No space constraints or color figure charges

- Immediate publication on acceptance

- Inclusion in PubMed, CAS, Scopus and Google Scholar

- Research which is freely available for redistribution 